\title{
Descriptively probabilistic relationship between mutated primary structure of von Hippel-Lindau protein and its clinical outcome
}

\author{
Shao-Min $\mathrm{Yan}^{1}$, Guang $\mathrm{Wu}^{2^{*}}$ \\ ${ }^{1}$ National Engineering Research Center for Non-food Biorefinery, Guangxi Academy of Sciences, 98 Daling Road, Nanning, Guangxi \\ Province, CN-530007, China; ${ }^{2}$ Computational Mutation Project, DreamSciTech Consulting, 301, Building 12, Nanyou A-zone, Jiannan \\ Road, Shenzhen, Guangdong Province CN-518054, China; *Corresponding author (hongguanglishibahao@yahoo.com), Tel: +86 771 \\ 2503 930, Fax: +86 75526648177.
}

Received 5 August 2008; revised 4 January 2009; accepted 7 January 2009.

\begin{abstract}
In this study, we use the cross-impact analysis to build a descriptively probabilistic relationship between mutant von Hippel-Lindau protein and its clinical outcome after quantifying mutant von Hippel-Lindau proteins with the amino-acid distribution probability, then we use the Bayesian equation to determine the probability that the von Hippel-Lindau disease occurs under a mutation, and finally we attempt to distinguish the classifications of clinical outcomes as well as the endocrine and nonendocrine neoplasia induced by mutations of von Hippel-Lindau protein. The results show that a patient has 9/10 chance of being von Hippel-Lindau disease when a new mutation occurs in von HippelLindau protein, the possible distinguishing of classifications of clinical outcomes using modeling, and the explanation of the endocrine and nonendocrine neoplasia in modeling view.
\end{abstract}

Keywords: Amino Acid; Bayes' Law; Cross-Impact Analysis; Distribution Probability; Mutation; Von Hippel-Lindau Disease

\section{INTRODUCTION}

Perhaps, the first step to study the genotype-phenotype relationship is to determine a protein in relation to a disease, and the second step would be to build a quantitative relationship between mutant protein and its clinical outcome. Then we may be in the position to predict the clinical outcome based on such a quantitative relationship, even to predict new functions led by new mutations.

Thus, we need the methods, which can quantify a protein sequence as a numeric sequence in order to build a quantitative relationship. In fact, we have various ways to quantify a protein sequence, for example, to use the physicochemical property of amino acid to quantify a protein sequence [1].

Since 1999, we have developed three approaches to quantify each amino acid in a protein as well as a whole protein (for reviews, see [2,3,4]), and our quantifications indeed differ before and after mutation, thus it is possible to use our approaches to build a quantitative relationship between changed primary structure and changed function of protein.

In 1911 and 1926, von Hippel and Lindau described the von Hippel-Lindau disease [5,6], later on Melmon and Rosen established the notion of the von HippelLindau disease [7], which is an autosomal dominant disorder characterized by cerebellar, spinal cord, and retinal hemangioblastomas; cysts of the kidney, pancreas, liver, and epididymis; and has an increased frequency of renal cancer (renal cell carcinoma or hypernephroma), pancreatic cancer, and pheochromocytoma $[8,9,10]$. The von Hippel-Lindau disease has a birth incidence of about 1 in 36000 and about $20 \%$ of cases arise as de novo mutations without a family history [11,12].

The von Hippel-Lindau disease tumor suppressor gene was identified in 1993 [13], of which mutations are the major cause for developing the von Hippel-Lindau disease. Pathologically relevant is inactivation of the von Hippel-Lindau gene and subsequent loss of the function of the von Hippel-Lindau protein, and Elongin B, C complex [14,15]. The dysfunction of the ubiquitination of hypoxia-inducible factors is an important step in the development of various tumors $[15,16,17,18,19]$. Also, a recent study elucidated the role of NGF/JunB/ EglN3related pathways in developmental apoptosis linking to tumourigenesis [20].

Clinically the von Hippel-Lindau disease is classified into two types: type I without pheochromocytoma and type II with pheochromocytoma [10,17]. On the other hand, more than 300 different von Hippel-Lindau muta- 
tions have been described at DNA level [21,22,23,24], and more than 100 at protein level. It would be great helpful if we can build a quantitative relationship between von Hippel-Lindau protein mutation and von Hippel-Lindau disease status, that is, the relationship between mutant protein and its clinical outcome.

In this study, we build a descriptively quantitative relationship between changed primary structure of mutated von Hippel-Lindau protein and the classification of its clinical outcome, distinguish the classifications of clinical outcomes as well as the endocrine and nonendocrine neoplasia induced by mutations of von Hippel-Lindau protein.

\section{MATERIALS AND METHODS}

\subsection{Data}

The human von Hippel-Lindau disease tumor suppressor with total 132 mutations (accession number P40337; December 4, 2007; Entry version 91) is obtained from UniProtKB/Swiss-Prot entry [25]. Among them, 123 are missense point mutations, 7 deletions and 3 insertions.

\subsection{Amino-Acid Distribution Probability}

Among three approaches developed by us, the amino-acid distribution probability is mainly related to the positions of amino acids along the protein, which is suitable for mutation analysis, and we have used this approach in a number of our previous studies [2,3,4,26,27,28,29,30,31,32,33,34,35,36,37, $38,39,40,41,42,43,44]$. The quantification is developed along such a thought, for example, how do two amino acids distribute along a protein sequence? Our intuition may suggest that there would be one amino acid in the first half of the sequence and another one in the second half. In fact, there are only three possible distributions, 1) both amino acids are in the first half, 2) one amino acid is in each half and 3) both amino acids are in the second half. Thus, each distribution has the probability of $1 / 3$. If we do not distinguish either the first half or second half but are simply interested in whether both amino acids are in both halves or in any half, there will be the probability of $1 / 2$ for each distribution.

If we are interested in the distribution probability of three amino acids in a protein, we naturally imagine to grouping the protein into three partitions, and our intuition may suggest that each partition contains an amino acid. If we do not distinguish the first, second and third partition, actually there are totally three types of distributions, i.e. 1) each amino acid is in each partition, 2) two amino acids are in a partition and an amino acid is in another partition, and 3) three amino acids are in a partition.

In this situation, the distribution probability can be calculated according to the statistical mechanics, which classifies the distribution of elementary particles in energy states according to three assumptions of whether distinguishing each particle and energy state, i.e. Maxwell-Boltzmann, Fermi-Dirac and Bose-Einstein as- sumptions [45]. We actually use the Maxwell-Boltzmann assumption for computing amino-acid distribution probability, which is equal to $\frac{r !}{q_{0} ! \times q_{1} ! \times \ldots \times q_{n} !} \times$ $\frac{r !}{r_{1} ! \times r_{2} ! \times \ldots \times r_{n} !} \times n^{-r}$ [45], where $r$ is the number of amino acids, $n$ is the number of partitions, $r_{n}$ is the number of amino acids in the $n$-th partition, $q_{n}$ is the number of partitions with the same number of amino acids, and ! is the factorial function.

Thus, the distribution probabilities are different for these three types of distributions of three amino acids, say, 0.2222 for 1 ), 0.6667 for 2) and 0.1111 for 3). Clearly the protein can only adopt one type of distribution for these three amino acids, which is the actual distribution probability.

For four amino acids, there are five distributions, 1) each partition contains an amino acid, 2) a partition contains two amino acids and two partitions contain an amino acid each, 3) two partitions contain two amino acids each, 4) a partition contains an amino acid and a partition contains three amino acids, and 5) a partition contains four amino acids. Their distribution probabilities are 0.0938 for 1 ), 0.5625 for 2), 0.1406 for 3), 0.1875 for 4 ), and 0.0156 for 5). Furthermore, there are seven distributions for five amino acids, 11 distributions for six amino acids, 15 distributions for seven amino acids, and so on.

\subsection{Quantification of Wild-Type von Hippel- Lindau Protein}

Table 1. Amino acids, their composition and distribution probability in wild-type human von Hippel-Lindau protein. (A, alanine; R, arginine; N, asparagine; D, aspartic acid; C, cysteine; E, glutamic acid; Q, glutamine; G, glycine; H, histidine; I, isoleucine; L, leucine; $\mathrm{K}$, lysine; $\mathrm{M}$, methionine; $\mathrm{F}$, phenylalanine; $\mathrm{P}$, proline; $\mathrm{S}$, serine; $\mathrm{T}$, threonine; $\mathrm{W}$, tryptophan; $\mathrm{Y}$, tyrosine; V, valine.)

\begin{tabular}{ccc}
\hline Amino acid & Number & Distribution probability \\
\hline A & 10 & 0.0476 \\
R & 20 & 0.0067 \\
N & 9 & 0.1770 \\
D & 11 & 0.1077 \\
C & 2 & 0.5000 \\
E & 30 & 0.0001 \\
Q & 8 & 0.0673 \\
G & 18 & 0.0389 \\
H & 5 & 0.0640 \\
I & 6 & 0.1543 \\
L & 20 & 0.0422 \\
K & 3 & 0.1111 \\
M & 3 & 0.6667 \\
F & 5 & 0.2880 \\
P & 19 & 0.0319 \\
S & 11 & 0.0404 \\
T & 7 & 0.2142 \\
W & 3 & 0.6667 \\
Y & 6 & 0.2315 \\
V & 17 & 0.1280 \\
\hline
\end{tabular}


With respect to the wild-type von Hippel-Lindau protein, for example, there are eight glutamines "Q" in von Hippel-Lindau protein (Table 1). We may ask how these eight Qs distribute along the von Hippel-Lindau protein? According to the problem of the occupancy of subpopulations and partitions [45], the simple way to answer this question is to imagine that we would divide the von Hippel-Lindau protein into eight equal partitions, and each partition has about 27 amino acids (213/8=26.625) be- cause the von Hippel-Lindau protein is composed of 213 amino acids, then there would be 22 configurations for all the possible distributions of eight Qs (Table 2).

Here, we calculate two distribution probabilities in Table 2 as example according to the above equation. For eight Qs equally distribute in each partition (the second row in Table 2), we have $q_{0}=0, q_{1}=8, \ldots q_{8}=0$; and $r_{1}=1$, $r_{2}=1, \ldots r_{8}=1$. Thus, we have the distribution probability,

$$
\begin{aligned}
& \frac{8 !}{0 ! \times 8 ! \times 0 ! \times 0 ! \times 0 ! \times 0 ! \times 0 ! \times 0 ! \times 0 !} \times \frac{8 !}{1 ! \times 1 ! \times 1 ! \times 1 ! \times 1 ! \times 1 ! \times 1 ! \times 1 !} \times 8^{-8} \\
& =\frac{40320}{1 \times 40320 \times 1 \times 1 \times 1 \times 1 \times 1 \times 1 \times 1} \times \frac{40320}{1 \times 1 \times 1 \times 1 \times 1 \times 1 \times 1 \times 1} \times \frac{1}{16777216}=0.0024
\end{aligned}
$$

Clearly, the von Hippel-Lindau protein can adopt only one distribution pattern, which is that two partitions contain zero $\mathrm{Q}$, five partitions contain one $\mathrm{Q}$ and one partition contains three Qs (the fourth row in Table 2).

$$
\begin{aligned}
& \frac{8 !}{2 ! \times 5 ! \times 0 ! \times 1 ! \times 0 ! \times 0 ! \times 0 ! \times 0 ! \times 0 ! \times 0 !} \times \frac{8 !}{0 ! \times 0 ! \times 1 ! \times 1 ! \times 1 ! \times 1 ! \times 1 ! \times 3 !} \times 8^{-8} \\
& =\frac{40320}{2 \times 120 \times 1 \times 1 \times 1 \times 1 \times 1 \times 1 \times 1} \times \frac{40320}{1 \times 1 \times 1 \times 1 \times 1 \times 1 \times 1 \times 6} \times \frac{1}{16777216}=0.0673
\end{aligned}
$$

In such a manner, we can quantify each amino acid in wild-type von Hippel-Lindau protein. Thereafter, we can assign these probabilities to each amino acid in the von Hippel-Lindau protein as shown in Figure 1, from which we get the visual sense of how these distribution probabilities go along the von Hippel-Lindau protein, and more importantly we can sum up these distribution probabilities together for all 213 amino acids in the protein.

Actually, the Maxwell-Bolzmann assumption provides
So we have $q_{0}=2, q_{1}=5, q_{2}=0, q_{3}=1, q_{4}=0, q_{5}=0, q_{6}=0$,

\begin{tabular}{|c|c|c|c|c|c|c|c|c|}
\hline Partition 1 & Partition 2 & Partition 3 & Partition 4 & Partition 5 & Partition 6 & Partition 7 & Partition 8 & Probability \\
\hline \multirow[t]{20}{*}{1} & 1 & 1 & 1 & 1 & 1 & 1 & 1 & 0.002403 \\
\hline & 1 & 1 & 1 & 1 & 1 & 1 & 2 & 0.0673 \\
\hline & & 1 & 1 & 1 & 1 & 1 & 3 & 0.0673 \\
\hline & & & & 1 & 1 & 1 & 5 & $5.6076 \mathrm{e}-3$ \\
\hline & & & & & 1 & 1 & 6 & $5.6076 \mathrm{e}-4$ \\
\hline & & & & & & 1 & 7 & $2.6703 e-5$ \\
\hline & & & & & & & 8 & $4.7684 \mathrm{e}-7$ \\
\hline & & 1 & 1 & 1 & 1 & 2 & 2 & 0.2523 \\
\hline & & & 1 & 1 & 1 & 2 & 3 & 0.2243 \\
\hline & & & & 1 & 1 & 2 & 4 & 0.0421 \\
\hline & & & & & 1 & 2 & 5 & $3.3646 \mathrm{e}-3$ \\
\hline & & & & & & 2 & 6 & $9.3460 \mathrm{e}-5$ \\
\hline & & & 1 & 1 & 2 & 2 & 2 & 0.1682 \\
\hline & & & & 1 & 2 & 2 & 3 & 0.0841 \\
\hline & & & & & 2 & 2 & 4 & $4.2057 \mathrm{e}-3$ \\
\hline & & & & 1 & 1 & 3 & 3 & 0.0280 \\
\hline & & & & & 2 & 3 & 3 & $5.6076 \mathrm{e}-3$ \\
\hline & & & & & 1 & 3 & 4 & $5.6076 \mathrm{e}-3$ \\
\hline & & & & & & 4 & 4 & $1.1683 \mathrm{e}-4$ \\
\hline & & & & & & 3 & 5 & $1.8692 \mathrm{e}-4$ \\
\hline
\end{tabular}
$q_{7}=0, q_{8}=0$; and $r_{1}=0, r_{2}=0, r_{3}=1, r_{4}=1, r_{5}=1, r_{6}=1, r_{7}=1$, $r_{8}=3$, that is,

Table 2. All possible distributions of eight glutamines in von Hippel-Lindau protein. (Bold and italic is the real distribution.)

us a way to estimate the position of amino acid in a protein, because there is a standard method for the computation using Maxwell-Bolzmann assumption, which saves us from inventing new computational methods. Moreover, the primary structure is the base for higher- level structure, thus any mutation in primary structure would lead to the change in distribution probability, in higher-level structure, and finally the biological function. This is the biological meaning of use of Maxwell- Bolzmann assumption for quantify- 


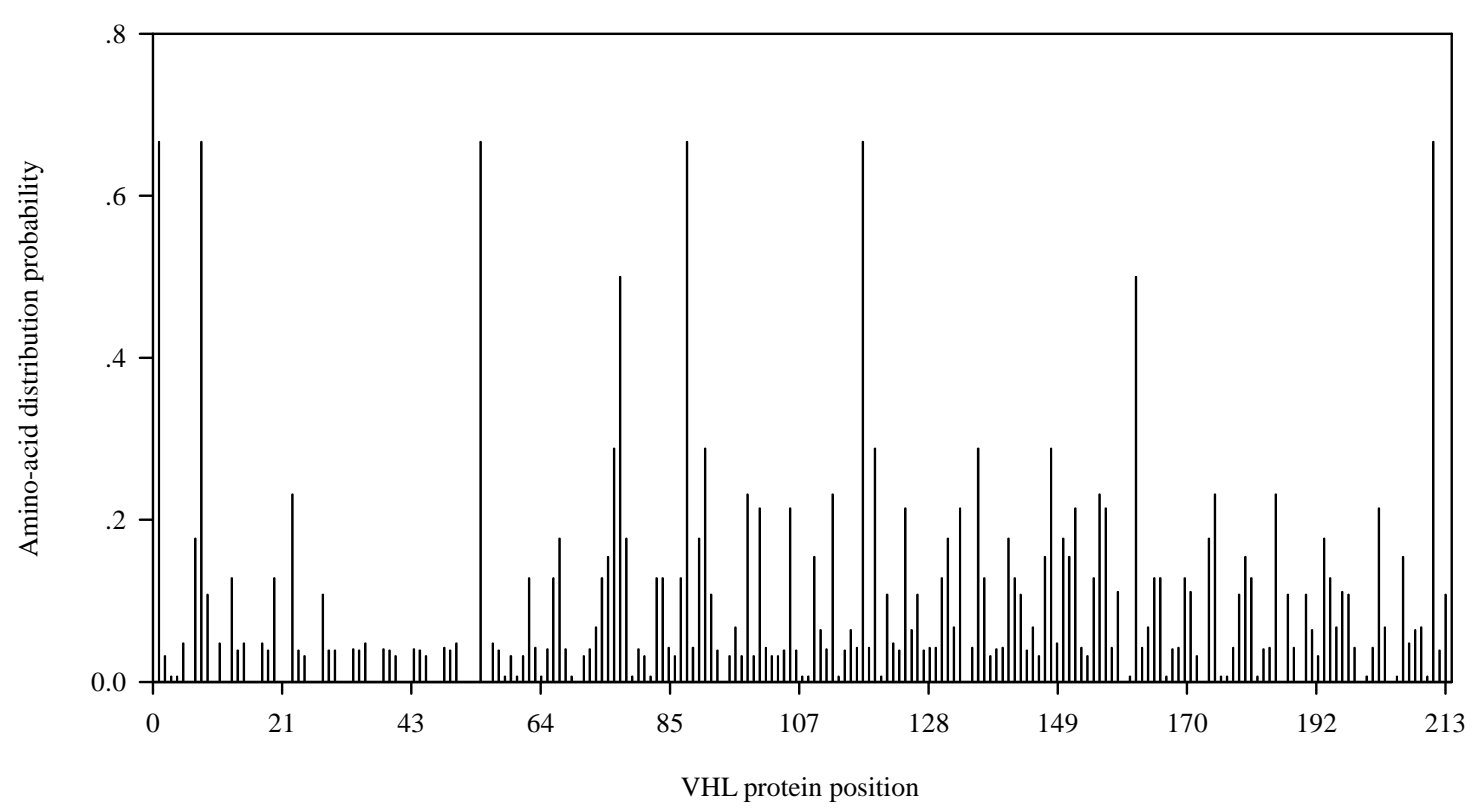

Figure 1. Visualization of amino-acid distribution probability in wild-type human von Hippel-Lindau protein.

cation of protein sequence.

In this context, any clinical manifestations related to mutation in proteins would have different distribution probabilities determined by Maxwell-Bolzmann assumption. This is the association between them.

\subsection{Quantification of Mutated von Hippel-Lindau Proteins}

The calculation in the above subsection is referred to the amino-acid distribution probability before mutation, say, the amino-acid distribution probability in wild-type von Hippel-Lindau protein. Obviously any point mutation leads an amino acid to change to another one, which certainly would change the distribution pattern of both original and mutated amino acids, thus the amino-acid distribution probability would differ for both original and mutated amino acids between before and after mutation.

For example, the missense mutations at the CpG mutation hotspot at codon 167 can mutate arginine "R" to glycine "G", or glutamine "Q" or tryptophan "W" [13, 46] leading to type I-II, type II and type II von HippelLindau disease, respectively. In above subsection, we have calculated the distribution probability of Qs (Table 2) before mutation, and now we show the calculation of distribution probability after R167Q mutation.

After this mutation, there are nine Qs in the von Hippel-Lindau mutant (Table 3), for which we have

$$
\frac{9 !}{5 ! \times 1 ! \times 1 ! \times 2 ! \times 0 ! \times 0 ! \times 0 ! \times 0 ! \times 0 ! \times 0 !} \times \frac{9 !}{0 ! \times 0 ! \times 0 ! \times 2 ! \times 0 ! \times 1 ! \times 3 ! \times 0 ! \times 3 !} \times 9^{-9}=0.0197
$$

while its distribution probability before this mutation is 0.0673 , so the mutation decreases the distribution probability of Q. On the other hand, there are 20 and 19 Rs before and after this mutation. Their distribution probabilities are 0.0067 and 0.0030 before and after mutation, so this mutation decreases the distribution probability of $\mathrm{R}$, too. The overall effect for this mutation is $(0.0030-0.0067)+(0.0197-0.0673)=-0.0513$, that is, the mutation reduces the distribution probability for von Hippel-Lindau protein.

Since von Hippel-Lindau protein functions as whole, we can calculate the change led by the mutation in following way. The sum of all the distribution probability is 19.6114 in wide-type von Hippel-Lindau protein (Figure 1), while the above calculated mutation leads the sum of all the distribution probability to be 19.1731 , thus this mutation results in $2.23 \%$ decrease in the measure [(19.1731-19.6114)/19.6114\%].

In this way, we have the quantitative measure for the changed primary structure of von Hippel-Lindau mutants and we also have documented clinical manifestations induced by the mutations of von Hippel-Lindau protein, thus we can build a quantitative relationship between changed structure and clinical outcome.

\subsection{Descriptively Probabilistic Relationship}

For building quantitative relationship between mutation and clinical outcome, we use the descriptively probabilistic method, as our quantification is the amino-acid distribution probability and each individual mutation related to its clinical outcome is presented as frequency. Therefore, we use the cross-impact analysis to couple 
Table 3. Distribution pattern of glutamines before and after mutation at position 167 in von Hippel-Lindau protein.

\begin{tabular}{lccccccccc}
\hline \multicolumn{1}{c}{ Partition } & I & II & III & IV & V & VI & VII & VIII & IX \\
\hline Before mutation & 0 & 0 & 1 & 1 & 1 & 1 & 1 & 3 & - \\
After mutation & 0 & 0 & 0 & 2 & 0 & 1 & 3 & 0 & 3 \\
\hline
\end{tabular}

them [35,47,48,49,50,51,52,53], because the amino-acid distribution probability either increases or decreases after mutation, which is a 2-possibilty event, and the clinical outcome either occurs or does not occur after mutation, which is a yes-and-no event. Thereafter, we can use the Bayesian equation to calculate the probability of occurrence of clinical outcome under a mutation.

\subsection{Classification of Clinical Outcomes}

It is extremely challenging how to use a mathematical modeling to distinguish the clinical outcomes with respect to mutant von Hippel-Lindau protein because of the variety of clinical outcomes. In an effort towards solving this problem, we employ our second quantification, amino-acid pair predictability, whose relational and applications have been published intensively (for reviews, see [2,3,4]).

This quantification is based on permutation, and can be calculated in the following way. For example, there are 30 glutamic acids " $E$ " and 20 Rs in von HippelLindau protein, the predicted frequency of amino-acid pair ER would be $3(30 / 213 \times 20 / 212 \times 212=2.817)$, while we do find three ERs in the protein, so the amino- acid pair ER is predictable. Still, the predicted frequency of EE would be $4(30 / 213 \times 29 / 212 \times 212=4.085)$, but actually the EE appears nine times in reality. This is the case that the actual frequency is larger than its predicted one. In this manner, we can quantify a protein sequence according to the percentage of how many amino-acid pairs are predictable among all the amino-acid pairs in given protein as well as its mutants. For instance, the predictable portion of amino-acid pairs is $27.54 \%$ in wild-type von Hippel-Lindau protein and 31.88\% in its P25L mutant.

\subsection{Statistics}

The data are presented as mean \pm SD for normal distribution or median with interquatile range for non-normal distribution. The Kruskal-Wallis one-way ANOVA and Chi-square are used for statistical inference, and $\mathrm{P}<0.05$ is considered significant.

\section{RESULTS AND DISCUSSION}

After computing amino-acid distribution probability in wild-type von Hippel-Lindau protein and in its $132 \mathrm{mu}-$ tants, we have 132 changed amino-acid distribution probabilities. Firstly, we can use the cross-impact analysis to build a quantitative relationship between the increase/decrease of distribution probability after muta- tions and the clinical diagnosis, because the cross-impact analysis is particularly suited for two relevant events coupled together [35,47,48,49,50,51,52,53].

Figure 2 displays the cross-impact analysis on the relationship between changed primary structure and von Hippel-Lindau disease. At the level of amino-acid distribution probability, $P(2)$ and $P(\overline{2})$ are the decreased and increased probabilities induced by mutations, and 53 and 79 mutations result in the distribution probability decreased and increased, respectively. At the level of clinical diagnosis: 1) $P(1 \mid \overline{2})$ is the impact probability (conditional probability) that the von Hippel-Lindau disease is diagnosed under the condition of increased distribution probability, and 70 mutations have such an effect. 2) $P(\overline{1} \mid \overline{2})$ is the impact probability that other disease is diagnosed under the condition of increased distribution probability, and 9 mutations work in such a manner. 3) $P(1 \mid 2)$ is the impact probability that the von Hippel-Lindau disease is diagnosed under the condition of decreased distribution probability, and 44 mutations play such a role. 4) $P(\overline{1} \mid 2)$ is the impact probability that other disease is diagnosed under the condition of decreased distribution probability, and 9 mutations fall into this category. At the level of combined events, we can see the combined results of changed structure and von Hippel-Lindau disease.

Table 4 lists the calculated probabilities with respect to Figure 2, from which several interesting points can be drawn. 1) As $P(\overline{2})$ is larger than $P(2)$, a mutation has a larger chance of increasing the distribution probability in von Hippel-Lindau mutant. 2) As $P(1 \mid \overline{2})$ is much larger than $P(\overline{1} \mid \overline{2})$, a mutation that increases the distribution probability has about nine tenth chance of being von Hippel-Lindau disease. 3) As $P(1 \mid 2)$ is much larger than $P(\overline{1} \mid 2)$, a mutation that decreases the distribution probability has much larger chance of being von HippelLindau disease.

Table 4. Computed probabilities in reference to the cross-impact analysis in Figure 2.

$$
\begin{aligned}
& P(2)=53 / 132=0.4015 \\
& P(\overline{2})=1-P(2)=1-0.4015=0.5985=79 / 132 \\
& P(1 \mid \overline{2})=70 / 79=0.8861 \\
& P(\overline{1} \mid \overline{2})=1-P(1 \mid \overline{2})=1-0.8861=0.1139=9 / 79 \\
& P(1 \mid 2)=44 / 53=0.8302 \\
& P(\overline{1} \mid 2)=1-P(1 \mid 2)=1-0.8302=0.1698=9 / 53 \\
& P(\overline{1})=P(1 \mid \overline{2}) \times P(\overline{2})=70 / 79 \times 79 / 132=0.5303=70 / 132 \\
& P(\overline{1} \overline{2})=P(\overline{1} \mid \overline{2}) \times P(\overline{2})=9 / 79 \times 79 / 132=0.0682=9 / 132 \\
& P(12)=P(1 \mid 2) \times P(2)=44 / 53 \times 53 / 132=0.3333=44 / 132 \\
& P(\overline{1} 2)=P(\overline{1} \mid 2) \times P(2)=9 / 53 \times 53 / 132=0.0682=9 / 132
\end{aligned}
$$




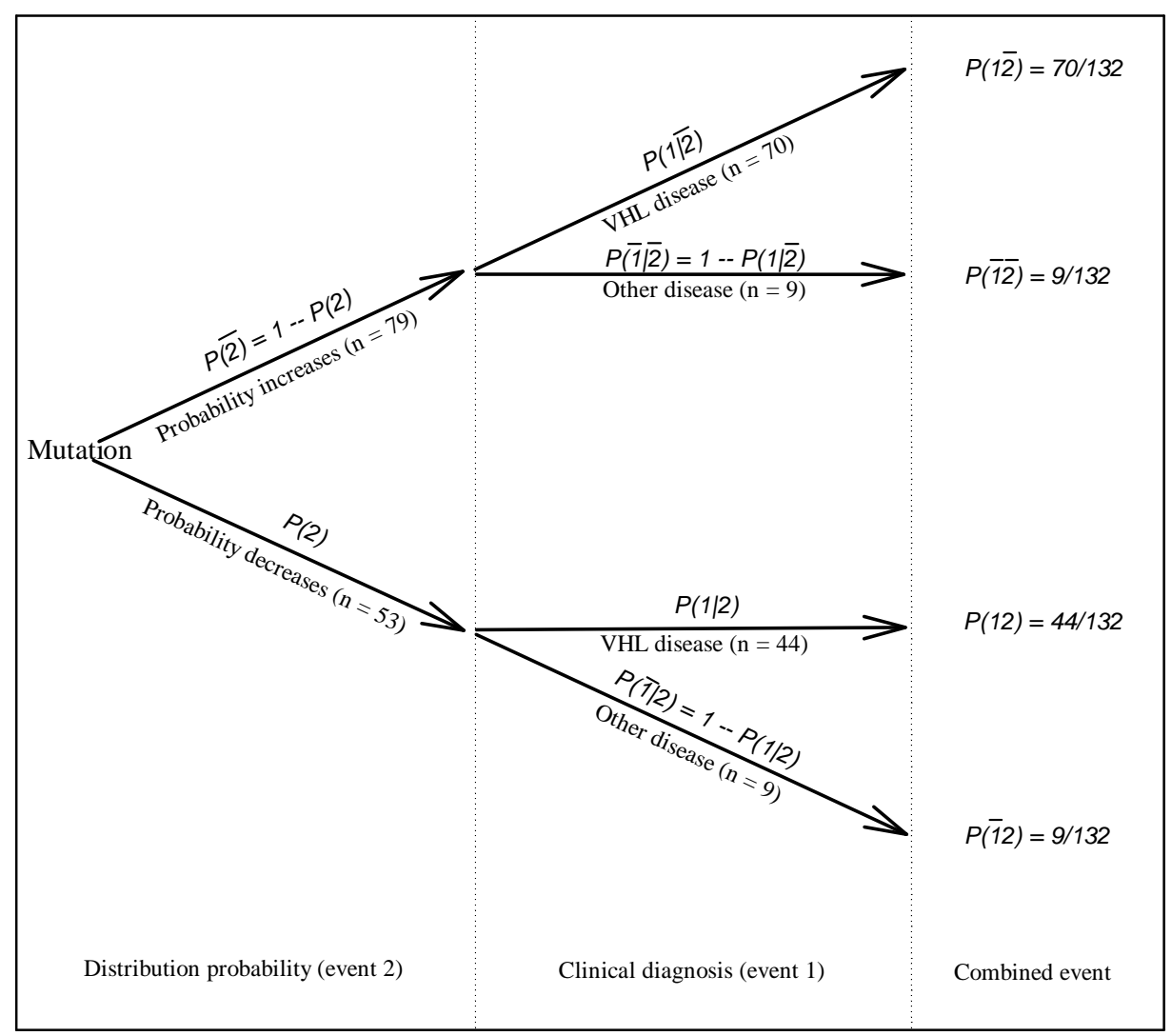

Figure 2. Cross-impact relationship among von Hippel-Lindau protein mutation, changed amino- acid distribution probability, and clinical diagnosis.

Secondly, we use the Bayes' law $P(1 \mid 2)=$ $P(2 \mid 1) \frac{P(1)}{P(2)}$, which indicates the probabilities of occurrences of two events [54], to determine the probability, $P(1)$, von Hippel-Lindau disease under a mutation, because $P(2)$ and $P(1 \mid 2)$ have already been defined in cross- impact analysis, while $P(2 \mid 1)$ is the probability that the distribution probability decreases under the condition that the von Hippel-Lindau disease is diagnosed.

As $P(1 \mid 2)=44 / 53=0.8302$ (Table 4), and $P(2 \mid 1)=44 /$

$(44+70)=0.3860, \quad P(1)=\frac{P(1 \mid 2)}{P(2 \mid 1)} P(2)=\frac{0.8302 \times 0.4015}{0.3860}$

$=0.8635$, namely, the patient has nine tenth chance of being von Hippel-Lindau disease when a new mutation is found in von Hippel-Lindau protein.

Among patients with von Hippel-Lindau disease, about $40 \%$ of mutations are genomic deletions and the rest are predominantly truncating or missense mutations, which do not occur within the first 53 amino acids $[55,56]$. In this study, we focus on the mutations of von Hippel-Lindau protein. From a probabilistic viewpoint, our results indicate the chance of being diagnosed as the von Hippel-Lindau disease when a new von Hippel-
Lindau mutant occurs.

The von Hippel-Lindau disease is characterized by marked phenotypic variability $[57,58]$, due to mosaicism [59], modifier effects [60], and mainly allelic heterogeneity [61]. All these result in complicated clinical classifications. Thus, we use the predictable portion of aminoacid pairs to model the classifications.

Figure 3 illustrates the classification with respect to the predictable portion of amino-acid pairs. Although there are large overlaps among classifications, our quantification already distinguishes them to some degree. For example, in comparison with von Hippel-Lindau disease, our quantification shows relatively lower in pheochromocytoma and higher in other disorders $(P=0.079$, Kruskal-Wallis one-way ANOVA). The lack of statistical significance is certainly, in part, due to few cases in some groups, however the trend is clear, which paves the way for further classification using more sophisticated mathematical models.

Genotype-phenotype relationships have revealed that a certain number of missense mutations are associated with a high risk of pheochromocytoma but the mutations that totally loss their functions are associated with a low risk. Most patients with type II von Hippel-Lindau disease have missense mutations whereas the large dele- 


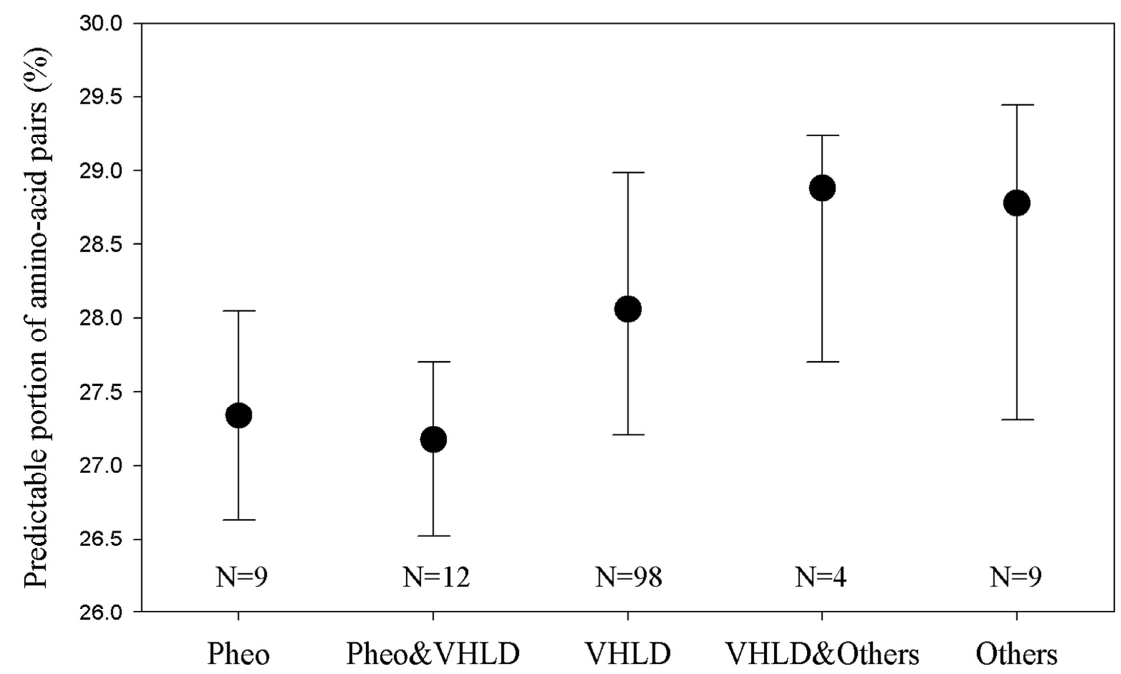

Figure 3. Predictable portion of amino-acid pairs induced by mutations of von Hippel-Lindau protein in pheochromocytoma (Pheo), von Hippel-Lindau disease and other disorders. The data are presented as median with an interquatile range $(P=0.079$, Kruskal-Wallis one-way ANOVA).

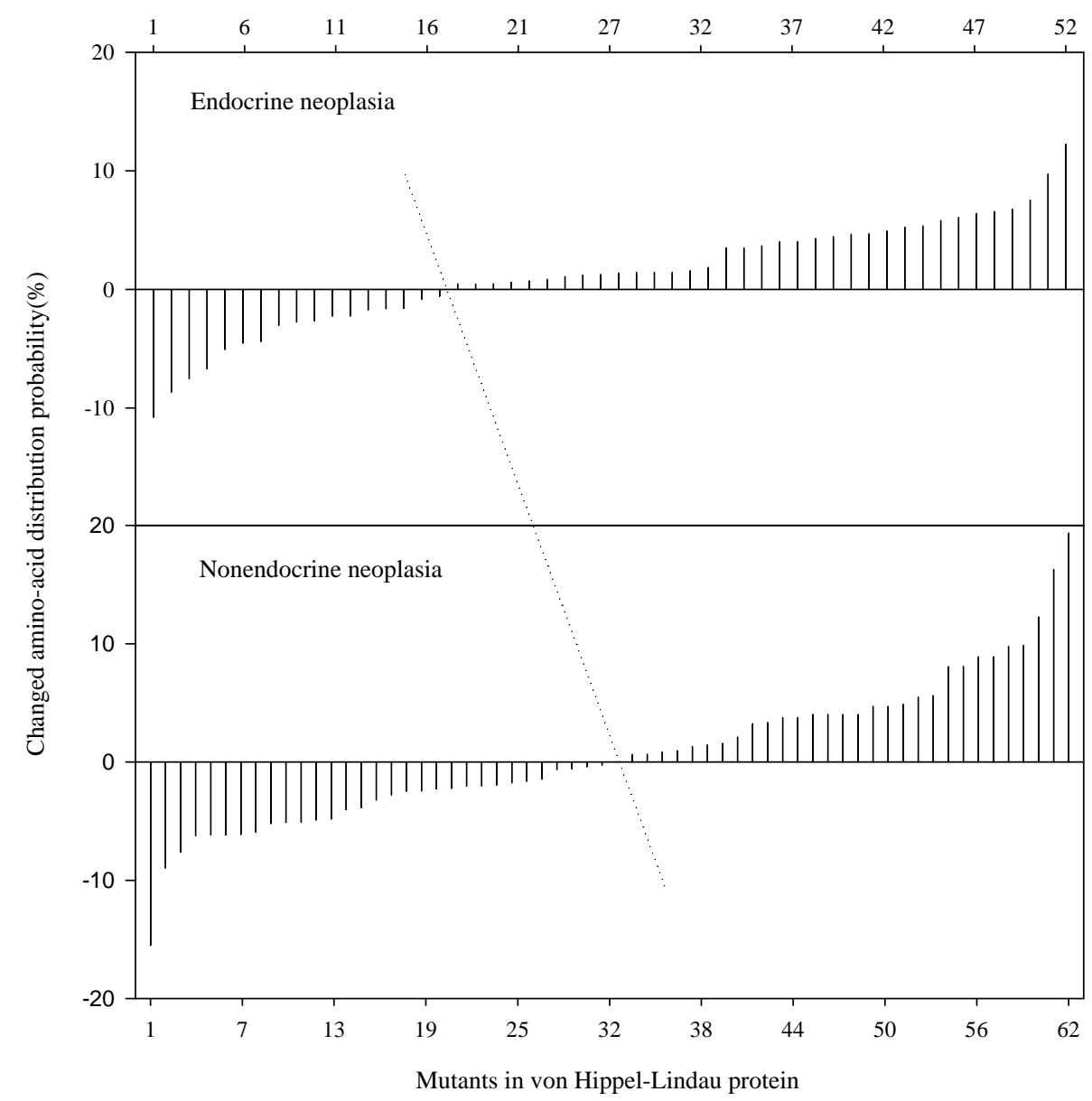

Figure 4. Distribution of changed amino-acid distribution probability in endocrine and nonendocrine neoplasia induced by mutations of von Hippel-Lindau protein ( $P=0.094$, Chi-square). 
tions and truncating mutations predominate in type I families [11,19,62,63]. Many missense mutations causing a type I phenotype are involved in the core hydrophobic residues and were predicted to disrupt protein structure, whereas type II phenotype missense mutations are involved in substitutions at a surface amino acid that does not cause a total loss of function [64,65].

Figure 4 displays the distribution of changed aminoacid distribution probability in endocrine neoplasia (pheochromocytoma, type II von Hippel-Lindau disease) and nonendocrine neoplasia (type I von Hippel-Lindau disease). As can be seen, the mutations that led to the endocrine neoplasia have the trend to increase the amino-acid distribution probability (upper panel), whereas the mutations that led to nonendocrine neoplasia have the effect to either increase or decrease the amino-acid distribution probability (lower panel). The difference between two panels is mainly considered from view of symmetry. As the x-axis is related to the number of von Hippel-Lindau mutations, this figure would be different when more mutations would be found in future, which might provide the much clearer pattern, although we did not find the statistical difference between two panels ( $P=0.094$, Chi-square) now.

From a theoretical viewpoint, one could consider to calculate the distribution probability of all 19 potential types of mutations at each position of von Hippel-Lindau protein, and then find the link between mutations and clinical outcomes. However, the amount of computation is huge because it would be equal to $2.369 \times 10^{272}$ mutations $\left(19^{213}\right)$, which is not only beyond the capacity of any computers, but also beyond the capacity for comparison. Actually, we really know that each position does not have 19 types of potential mutations, because this mutation process is governed by the translation probability between RNA codon and mutated amino acids [66, $67,68]$. On the other hand, our study is focused on the documented data rather than the simulated data.

In this study, we use a single value, the sum of all distribution probability to represent the normal von HippelLindau protein and its mutated proteins, respectively, because there is no other way to use a single value dynamically to represent a protein, namely, the value is different when a protein is different. Without such a measure, we cannot model a protein dynamically with its mutations. To the best of knowledge, currently it is only the accession number that can represent a protein uniquely, however it has nothing to do with the protein itself, i.e. composition, length, function, etc.

In general, one would hope to verify this type of study against the real-life cases, which is possible in future although it would deal with a large-scale collaboration because this type of diseases is not frequently seen in clinical settings, for example, the von Hippel-Lindau disease has a birth incidence of about 1 in 36000 [11,12].
It will take years to verify what a theoretical study finds with fast-speed computational technique. Even, we cannot verify all the theoretical studies, for example, we cannot create another earth without global warming.

The implications of this study include two aspects. 1) The relationship between changed primary structure and changed function is very meaningful, because it provides the dynamic rather than static relationship between mutant protein and its function. This can furthermore provide us the basis for building a dynamic model to predict the new function in mutant proteins. Nevertheless, we need to quantify the proteins in order to build a dynamic model and this study is doing in such a way. 2) From the clinical viewpoint, the classification of von HippelLindau disease as well as many mutation related diseases needs a considerable amount of clinical assays. Our approach can provide a probabilistic estimate for disease classification after determining which amino acid has mutated, because the primary structure of protein is the base for its high-level structure and function.

\section{ACKNOWLEDGEMENTS}

This study was partly supported by Guangxi Science Foundation (No. 0537012-G and 0991080), and Guangxi Academy of Sciences (project No. 09YJ17SW07).

\section{REFERENCES}

[1] K. C. Chou, (2004) Structure bioinformatics and its impact to biomedical science, Curr. Med. Chem, 11, 2105-2134.

[2] G. Wu and S. Yan, (2002) Randomness in the primary structure of protein: Methods and implications, Mol. Biol. Today, 3, 55-69.

[3] G. Wu and S. Yan, (2006) Mutation trend of hemagglutinin of influenza A virus: A review from computational mutation viewpoint, Acta Pharmacol. Sin., 27, 513-526.

[4] G. Wu and S. Yan, (2008) Lecture notes on computational mutation, Nova Science Publishers, New York, 2008.

[5] Von Hippel, (1911) Die anatomische Grund lage der von mir beschriebenen 'sehr seltenen Erkrankung der Netzhaut', Graefes. Arch. Ophthalmol., 79, 350-377.

[6] A. Lindau, (1926) Studien uber kleinhirncysten, bau, pathogenese und bezoejimgem zur angiomatosis retinae, Acta Pathol. Microbiol. Scand., Suppl 1, 1-128.

[7] K. L. Melmon and S. W. Rosen, (1964) Lindau's disease, Am. J. Med., 36, 595-617.

[8] V. V. Michels, (1988) Investigative studies in von Hippel-Lindau disease, Neurofibromatosis, 1, 159-163.

[9] H. P. Neumann, (1987) Basic criteria for clinical diagnosis and genetic counselling in von Hippel-Lindau syndrome, Vasa, 16, 220-226.

[10] R. R. Lonser, G. M. Glenn, M. Walther, E. Y. Chew, S. K. Libutti, W. M. Linehan, and E. H. Oldfield, (2003) von Hippel-Lindau disease, Lancet, 361, 2059-2067.

[11] E. R. Maher, A. R. Webster, F. M. Richards, J. S. Green, P. A. Crossey, S. J. Payne, and A. T. Moore, (1996) Phenotypic expression in von Hippel-Lindau disease: Corre- 
lations with germline VHL gene mutations, J. Med. Genet., 33, 328-332.

[12] F. M. Richards, S. J. Payne, B. Zbar, N. A. Affara, M. A. Ferguson-Smith, and E. R. Maher, (1995) Molecular analysis of de novo germline mutations in the von Hippel-Lindau disease gene, Hum. Mol. Genet., 4, 2139-2143.

[13] F. Latif, K. Tory, J. Gnarra, M. Yao, F. M. Duh, M. L. Orcutt, et al., (1993) Identification of the von Hippel-Lindau disease tumor suppressor gene, Science, 260, 1317-1320.

[14] P. O. Schnell, M. L. Ignacak, A. L. Bauer, J. B. Striet, W. R. Paulding, and M. F. Czyzyk-Krzeska, (2003) Regulation of tyrosine hydroxylase promoter activity by the von Hippel-Lindau tumor suppressor protein and hypoxia-inducible transcription factors, J. Neurochem., 85, 483-491.

[15] W. G. Jr. Kaelin, (2002) Molecular basis of the VHL hereditary cancer syndrome, Nat. Rev. Cancer, 2, 673-682.

[16] W. G. Jr. Kaelin, (2003) The von Hippel-Lindau gene, kidney cancer, and oxygen sensing, J. Am. Soc. Nephrol., 14, $2703-2711$.

[17] T. Shuin, I. Yamasaki, K. Tamura, H. Okuda, M. Furihata, and S. Ashida, (2006) Von Hippel-Lindau disease: Molecular pathological basis, clinical criteria, genetic testing, clinical features of tumors and treatment, Jpn. J. Clin. Oncol., 36, 337-343.

[18] M. Ohh, (2006) Ubiquitin pathway in VHL cancer syndrome, Neoplasia, 8, 623-629.

[19] F. Chen, T. Kishida, M. Yao, T. Hustad, D. Glavac, M. Dean, J. R. Gnarra, M. L. Orcutt, F. M. Duh, G. Glenn, J. Green, Y. E. Hsia, J. Lamiell, H. Li, M. H. Wei, L. Schmidt, K. Tory, I. Kuzmin, T. Stackhouse, F. Latif, W. M. Linehan, M. Lerman, and B. Zbar, (1995) Germline mutations in the von Hippel-Lindau disease tumor suppressor gene: Correlations with phenotype, Hum. Mutat., 5, 66-75.

[20] S. Lee, E. Nakamura, H. Yang, W. Wei, M. S. Linggi, M. P. Sajan, R. V. Farese, R. S. Freeman, B. D. Carter, W. G. Jr. Kaelin, and S. Schlisio, (2005) Neuronal apoptosis linked to EglN3 prolyl hydroxylase and familial phaeochromocytoma genes: developmental culling and cancer. Cancer Cell, 8, 1-13.

[21] Clinical Research Group for VHL in Japan, (1995) Germline mutations in the von Hippel-Lindau disease (VHL) gene in Japanese VHL, Hum. Mol. Genet., 4, 2233-2237.

[22] H. P. Neumann, B. Bender, I. Zauner, D. P. Berger, C. Eng, H. Brauch, and B. Zbar, (1996) Monogenetic hypertension and pheochromocytoma, Am. J. Kidney Dis., 28, 329-333.

[23] S. Olschwang, S. Richard, C. Boisson, S. Giraud, P. Laurent- Puig, F. Resche, and G. Thomas, (1998) Germline mutation profile of the VHL gene in von Hippel-Lindau disease and in sporadic hemangioblastoma, Hum. Mutat., 12, 424-430.

[24] C. Stolle, G. Glenn, B. Zbar, J. S. Humphrey, P. Choyke, M. Walther, S. Pack, K. Hurley, C. Andrey, R. Klausner, and W. M. Linehan, (1998) Improved detection of germ- line mutations in the von Hippel-Lindau disease tumor suppressor gene, Hum. Mutat., 12, 417-423.

[25] A. Bairoch and R. Apweiler, (2000) The SWISS-PROT protein sequence data bank and its supplement TrEMBL in 2000, Nucleic Acids Res., 28, 45-48.

[26] N. Gao, S. Yan, and G. Wu, (2006) Pattern of positions sensitive to mutations in human haemoglobin $\alpha$-chain, Protein Pept. Lett., 13, 101-107.

[27] G. Wu and S. Yan, (2000) Prediction of distributions of amino acids and amino acid pairs in human haemoglobin $\alpha$-chain and its seven variants causing-thalassemia from their occurrences according to the random mechanism, Comp. Haematol. Int, 10, 80-84.

[28] G. Wu and S. Yan, (2001) Analysis of distributions of amino acids, amino acid pairs and triplets in human insulin precursor and four variants from their occurrences according to the random mechanism, J. Biochem. Mol. Biol. Biophys., 5, 293-300.

[29] G. Wu and S. Yan, (2001) Analysis of distributions of amino acids and amino acid pairs in human tumor necrosis factor precursor and its eight variants according to random mechanism, J. Mol. Model, 7, 318-323.

[30] G. Wu and S. Yan, (2002) Random analysis of presence and absence of two-and three-amino-acid sequences and distributions of amino acids, two- and three-amino-acid sequences in bovine p53 protein, Mol. Biol. Today, 3, 31-37.

[31] G. Wu and S. Yan, (2002) Analysis of distributions of amino acids in the primary structure of apoptosis regulator Bcl-2 family according to the random mechanism, J. Biochem. Mol. Biol. Biophys, 6, 407-414.

[32] G. Wu and S. Yan, (2002) Analysis of distributions of amino acids in the primary structure of tumor suppressor p53 family according to the random mechanism, J. Mol. Model, 8, 191-198.

[33] G. Wu and S. Yan, (2004) Determination of sensitive positions to mutations in human p53 protein, Biochem. Biophys. Res. Commun., 321, 313-319.

[34] G. Wu and S. Yan, (2005) Searching of main cause leading to severe influenza A virus mutations and consequently to influenza pandemics/epidemics, Am. J. Infect. Dis., 1, 116-123.

[35] G. Wu and S. Yan, (2005) Prediction of mutation trend in hemagglutinins and neuraminidases from influenza A viruses by means of cross-impact analysis, Biochem. Biophys. Res. Commun., 326, 475-482.

[36] G. Wu and S. Yan, (2006) Timing of mutation in hemagglutinins from influenza A virus by means of amino-acid distribution rank and fast Fourier transform, Protein Pept. Lett., 13, 143-148.

[37] G. Wu and S. Yan, (2006) Prediction of possible mutations in H5N1 hemagglutinins of influenza A virus by means of logistic regression, Comp. Clin. Pathol., 15, 255-261.

[38] G. Wu and S. Yan, (2006) Prediction of mutations in H5N1 hemagglutinins from influenza A virus, Protein Pept. Lett., 13, 971-976.

[39] G. Wu and S. Yan, (2007) Improvement of model for prediction of hemagglutinin mutations in H5N1 influenza 
viruses with distinguishing of arginine, leucine and serine, Protein Pept. Lett., 14, 191-196.

[40] G. Wu and S. Yan, (2007) Improvement of prediction of mutation positions in H5N1 hemagglutinins of influenza A virus using neural network with distinguishing of arginine, leucine and serine, Protein Pept. Lett., 14, 465-470.

[41] G. Wu and S. Yan, (2007) Prediction of mutations engineered by randomness in H5N1 neuraminidases from influenza A virus, Amino Acids, 34, 81-90.

[42] G. Wu and S. Yan, (2007) Prediction of mutations in H1 neuraminidases from North America influenza A virus engineered by internal randomness, Mol. Divers., 11, 131-140.

[43] G. Wu and S. Yan, (2008) Prediction of mutations initiated by internal power in H3N2 hemagglutinins of influenza A virus from North America, Int. J. Pept. Res. Ther., 14, 41-51.

[44] G. Wu and S. Yan, (2008) Prediction of mutation in H3N2 hemagglutinins of influenza A virus from North America based on different datasets, Protein Pept. Lett., 15, 144-152.

[45] W. Feller, (1968) An introduction to probability theory and its applications, 3rd ed, Wiley, New York, 1, 34-40.

[46] B. Zbar, T. Kishida, F. Chen, L. Schmidt, E. R. Maher, F. M. Richards, P. A. Crossey, A. R. Webster, N. A. Affara, M. A. Ferguson-Smith, et al., (1996) Germline mutations in the Von Hippel-Lindau disease (VHL) gene in families from North America, Europe, and Japan, Hum. Mutat., 8, 348-357.

[47] T. G. Gordon and H. Hayward, (1968) Initial experiments with the cross-impact matrix method of forecasting, Futures, 1, 100-116.

[48] T. G. Gordon, (1969) Cross-impact matrices - an illustration of their use for policy analysis, Futures, 2, 527-531.

[49] S. Enzer, (1970) Delphi and cross-impact techniques: an effective combination for systematic futures analysis, Futures, 3, 48-61.

[50] S. Enzer, (1970) Cross-impact techniques in technology assessment, Futures, 4, 30-51.

[51] A. P. Sage, (1977) Methodology for large-scale systems, McGraw-Hill, New York, 165-203.

[52] G. Wu, (2000) Application of cross-impact analysis to the relationship between aldehyde dehydrogenase 2 and flushing, Alcohol Alcohol., 35, 55-59.

[53] G. Wu and S. Yan, (2008) Building quantitative relationship between changed sequence and changed oxygen affinity in human hemoglobin-chain, Protein Pept. Lett., 15, 341-345.

[54] Wikipedia, (2008) Bayes' theorem, http://en.wikipedia.org/wiki/ Bayes'_theorem.

[55] S. O. Ang, H. Chen, K. Hirota, V. R. Gordeuk, J. Jelinek, Y. Guan, E. Liu, A. I. Sergueeva, G. Y. Miasnikova, D. Mole, P. H. Maxwell, D. W. Stockton, G. L. Semenza, and J. T. Prchal., (2002) Disruption of oxygen homeostasis underlies congenital Chuvash polycythemia, Nature Genet., 32, 614-621.
[56] Y. Pastore, K. Jedlickova, Y. Guan, E. Liu, J. Fahner, H. Hasle, J. F. Prchal, and J. T. Prchal., (2003) Mutations of von Hippel- Lindau tumor-suppressor gene and congenital polycythemia, Am. J. Hum. Genet., 73, 412-419.

[57] E. R. Maher, (2004) Von Hippel-Lindau disease, Curr. Mol. Med., 4, 833-842.

[58] E. R. Woodward and E. R. Maher, (2006) Von Hippel-Lindau disease and endocrine tumour susceptibility, End. Relat. Cancer, 13, 415-425.

[59] M. T. Sgambati, C. Stolle, P. L. Choyke, M. M. Walther, B. Zbar, W. M. Linehan, and G. M. Glenn, (2000) Mosaicism in von Hippel-Lindau disease: lessons from kindreds with germline mutations identified in offspring with mosaic parents, Am. J. Hum. Genet., 66, 84-91.

[60] A. R. Webster, F. M. Richards, F. E. MacRonald, A. T. Moore, and E. R. Maher, (1998) An analysis of phenotypic variation in the familial cancer syndrome von Hippel-Lindau disease: evidence for modifier effects, Am. J. Hum. Genet., 63, 1025-1035.

[61] P. A. Crossey, C. Eng, M. Ginalska-Malinowska, T. W. J. Lennard, J. R. Sampson, B. A. J. Ponder, and E. R. Maher, (1995) Molecular genetic diagnosis of von Hippel-Lindau disease in familial phaeochromocytoma, J. Med. Genet., 32, 885-886.

[62] P. A. Crossey, F. M. Richards, K. Foster, J. S. Green, A. Prowse, F. Latif, M. I. Lerman, B. Zbar, N. A. Affara, M. A. Ferguson-Smith, and R. Maher, (1994) Buys CHCM, identification of intragenic mutations in the von Hippel-Lindau disease tumour suppressor gene and correlation with disease phenotype, Hum. Mol. Genet., 3, 1303-1308.

[63] E. R. Maher, A. R. Webster, F. M. Richards, J. S. Green, P. A. Crossey, S. J. Payne, and A. T. Moore, (2000) Phenotypic expression in von Hippel-Lindau disease: correlations with germline VHL gene mutations, J. Med. Genet., 37, 62-63.

[64] C. E. Stebbins, W. G. Jr. Kaelin, and N. P. Pavletich, (1999) Structure of the VHL-ElonginC-ElonginB complex: Implications for VHL tumor suppressor function, Science, 284, 455-461.

[65] S. J. Marx and W. F. Simonds, (2005) Hereditary hormone excess: Genes, molecular pathways, and syndromes, End. Rev., 26, 615-661.

[66] G. Wu and S. Yan, (2005) Determination of mutation trend in proteins by means of translation probability between RNA codes and mutated amino acids, Biochem. Biophys. Res. Commun., 337, 692-700.

[67] G. Wu and S. Yan, (2006) Determination of mutation trend in hemagglutinins by means of translation probability between RNA codons and mutated amino acids, Protein Pept. Lett., 13, 601-609.

[68] G. Wu and S. Yan, (2007) Translation probability between RNA codons and translated amino acids, and its applications to protein mutations, in: Leading-Edge Messenger RNA Research Communications, ed. Ostrovskiy M. H. Nova Science Publishers, New York, Chapter 3, 47-65. 